\title{
Reliable Interlayer Based on Hybrid Nanocomposites and Carbon Nanotubes for Lithium-Sulfur Batteries
}

Tao Liu, ${ }^{\dagger}$ Shimei Sun, ${ }^{\dagger}$ Jialiang Hao, ${ }^{\S}$ Wei Song, ${ }^{\prime \prime}$ Quanhai Niu, Xiaolin Sun, ${ }^{\dagger}$ Yue Wu, Depeng Song, and Jianfei $\mathrm{Wu}^{*+, \ldots}$

${ }^{\dagger}$ Qingdao Institute of Bioenergy and Bioprocess Technology, Chinese Academy of Sciences, Qingdao 266101, PR China

${ }^{\ddagger}$ Center of Materials Science and Optoelectronics Engineering, University of Chinese Academy of Sciences, Beijing 100049, PR China

\$Ocean University of China, Qingdao 266100, PR China

"School of Chemistry, Sun Yat-sen University, Guangzhou 510275, PR China

${ }^{*}$ Corresponding Authors

E-mail: wujf@qibebt.ac.cn (Jianfei Wu) 


\section{Making of oxidized CNTs (OCNT).}

CNTs (Tiannai (zhenjiang) Technology Co., Ltd ) were first purified by calcination at $550^{\circ} \mathrm{C}$ for $1 \mathrm{~h}$ and washing with diluted hydrochloric acid ${ }^{[1]}$. Afterwards, $500 \mathrm{mg}$ purified CNTs were refluxed in a round-bottomed flask which contains $60 \mathrm{~mL}$ concentrated sulfuric acid and $20 \mathrm{~mL}$ concentrated nitric acid at $80^{\circ} \mathrm{C}$ for $2 \mathrm{~h}$. The OCNTs were washed thoroughly by water and ethanol for several time, collected by centrifugation, and dried in a vacuum oven at $80{ }^{\circ} \mathrm{C}$ for $12 \mathrm{~h}$.

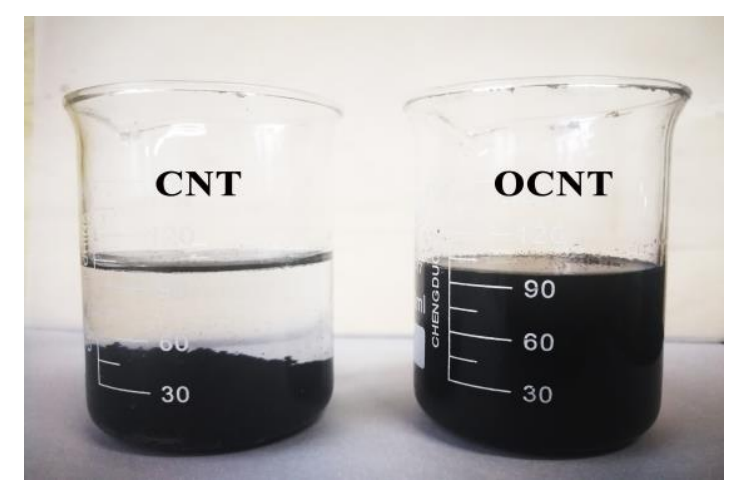

Figure S1. The photograph of raw CNT and OCNT dissolved in the mixed solution of ethylene glycol and water, followed by vigorous shaking and resting for $24 \mathrm{~h}$.

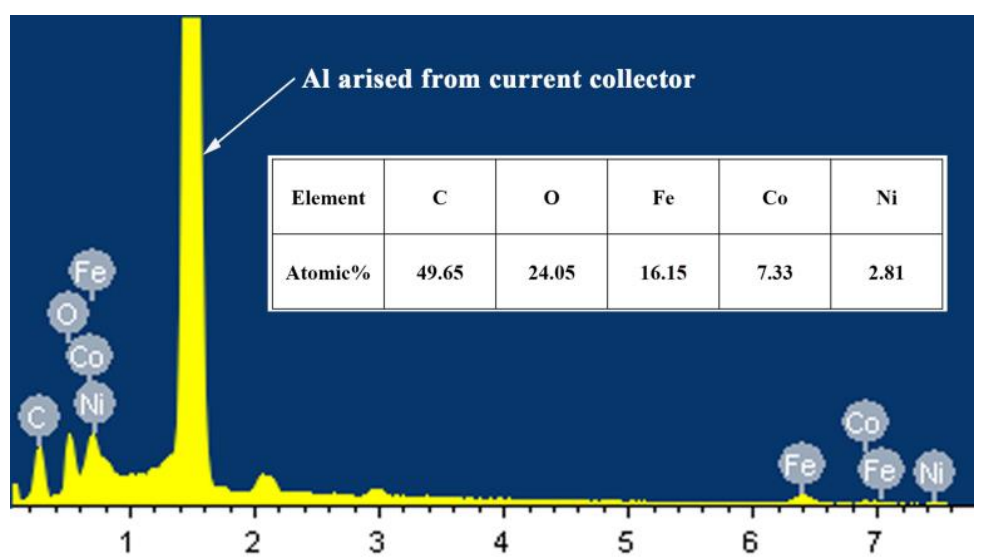

Figure S2. EDS spectra and molar ratio of the corresponding element of CNFO@CNT composite.
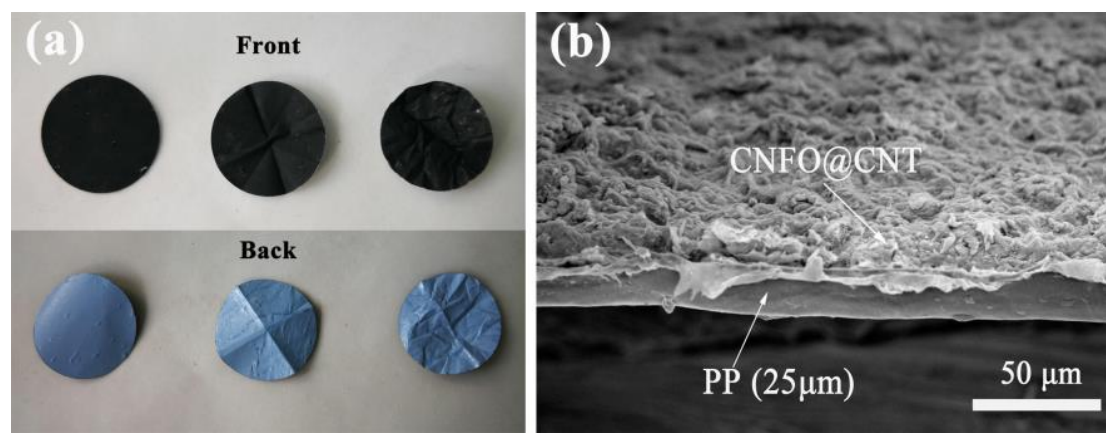

Figure S3. (a) Digital images of the folding/unfolding tests of CNFO@CNT-modified separator. (b) Cross-sectional SEM observation of CNFO@CNT/PP. 


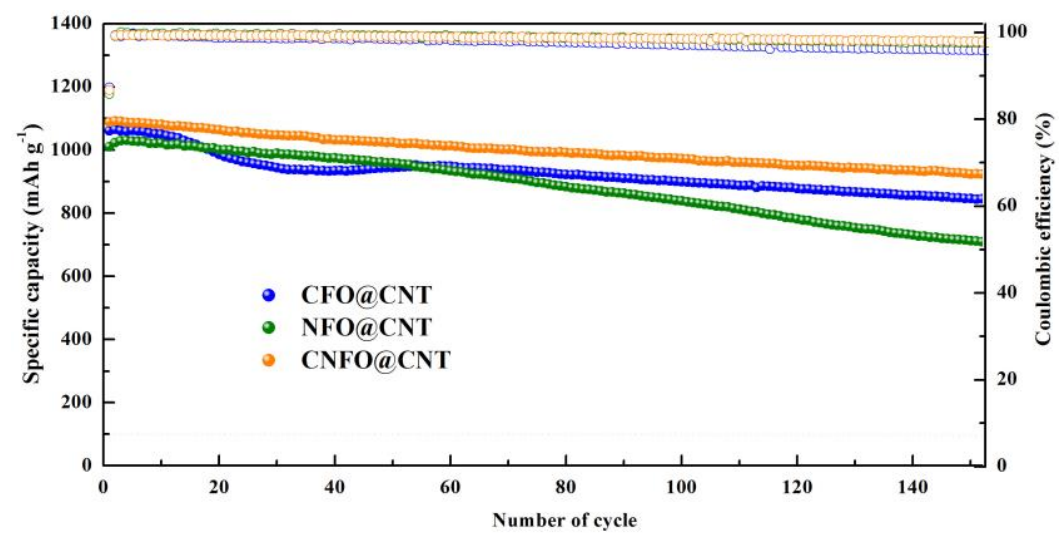

Figure S4. Cycling performance of the Li-S batteries using different interlayers at $1.0 \mathrm{C}$ with sulfur mass loading of 1.0 $\mathrm{mg} \mathrm{cm}{ }^{-2}$.
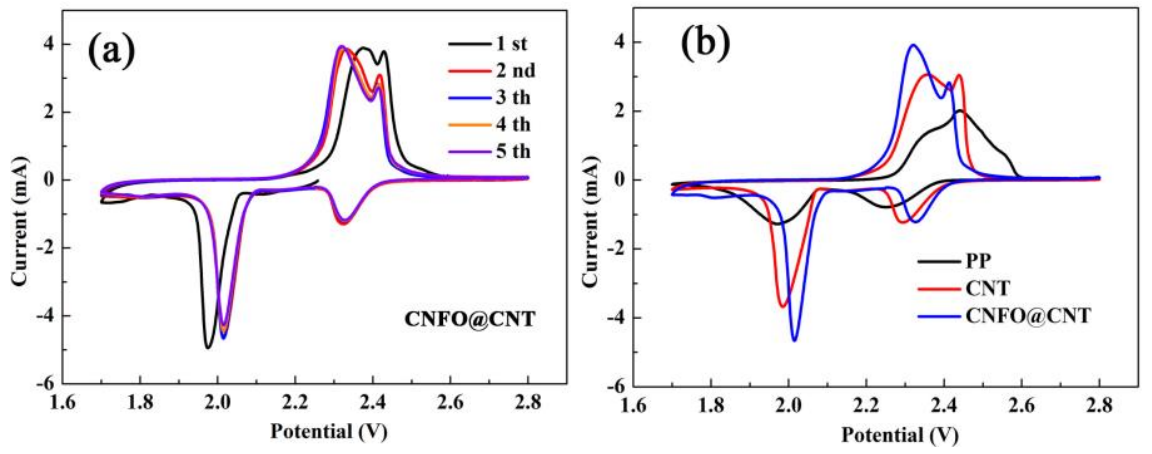

Figure S5. (a) Multi-cycle voltammograms of the Li-S batteries using CNFO@CNT interlayers, and (b) CV curves of the $\mathrm{Li}-\mathrm{S}$ batteries using different interlayers at a scan rate of $0.1 \mathrm{mV} \mathrm{s}^{-1}$.
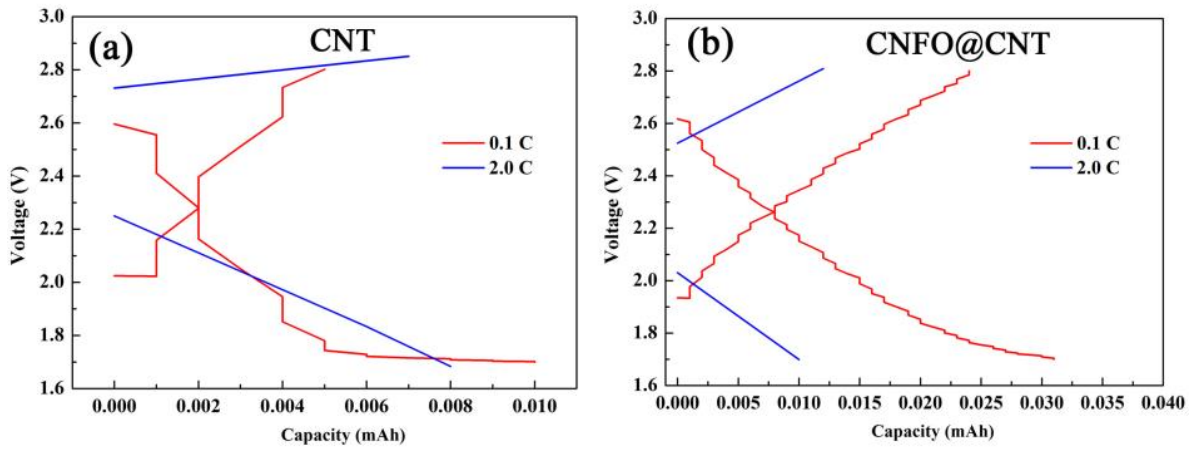

Figure S6. The capacitive contribution of the (a) CNT and (b) CNFO@CNT interlayers without any sulfur loading at 0.1 and $2.0 \mathrm{C}$. 


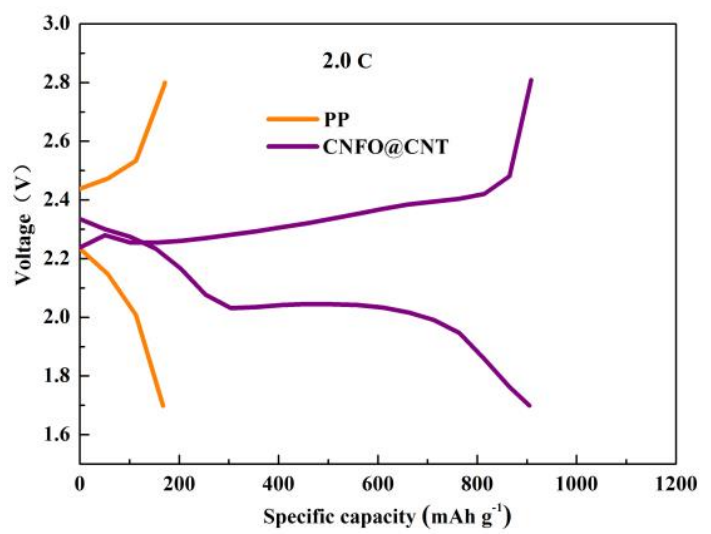

Figure S7. Discharge-charge curves of the Li-S batteries with PP and CNFO@CNT/PP at 2.0 C.
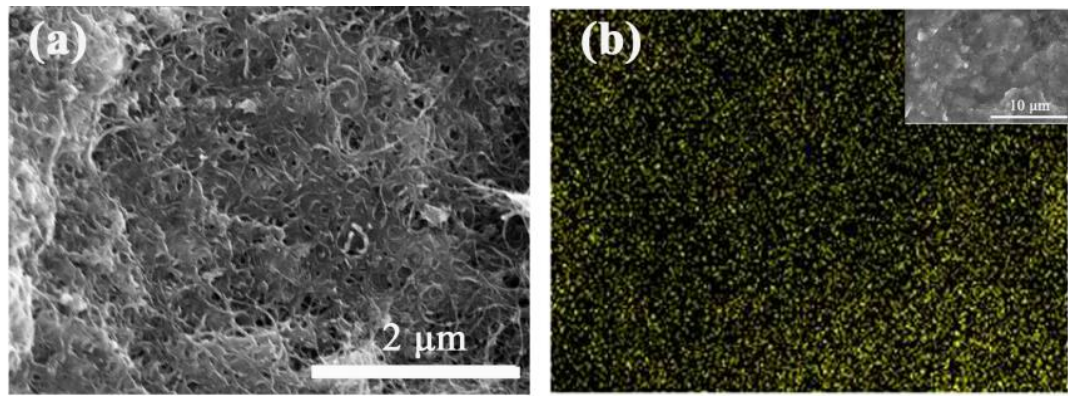

Figure S8. (a) SEM and (b) Elemental mapping images of the CNFO@CNT interlayers and corresponding SEM images (inset) on the cathode side after a single charge-discharge cycle.
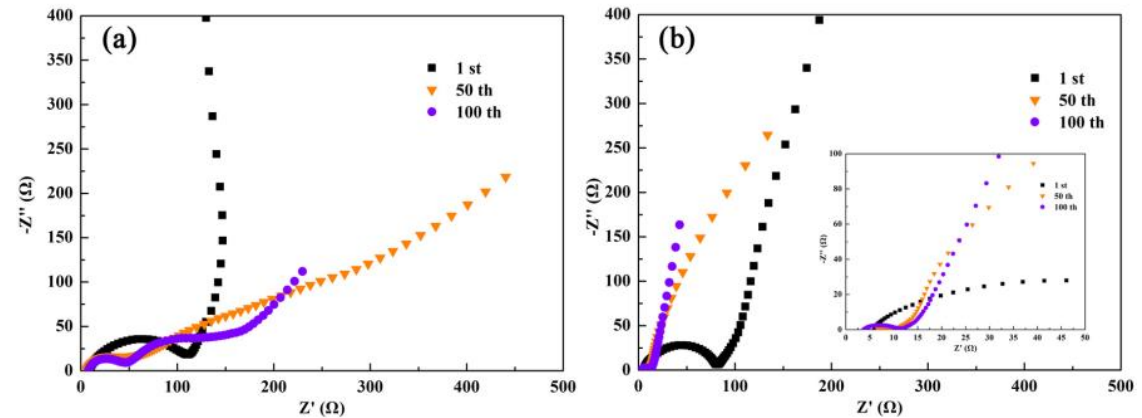

Figure S9. Impedance plots of Li-S batteries based on (a) PP-separator and (b) CNFO@CNT-separator, before cycling and after 50, 100 cycles. 


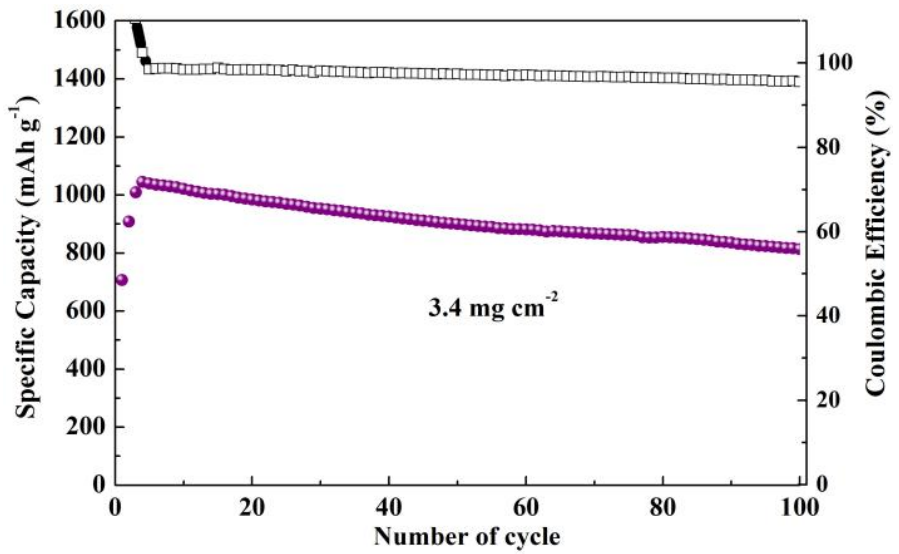

Figure S10. Cycling performance of Li-S batteries with CNFO@CNT/PP at $0.5 \mathrm{C}$ with the sulfur loading of $3.4 \mathrm{mg} \mathrm{cm}^{-2}$.
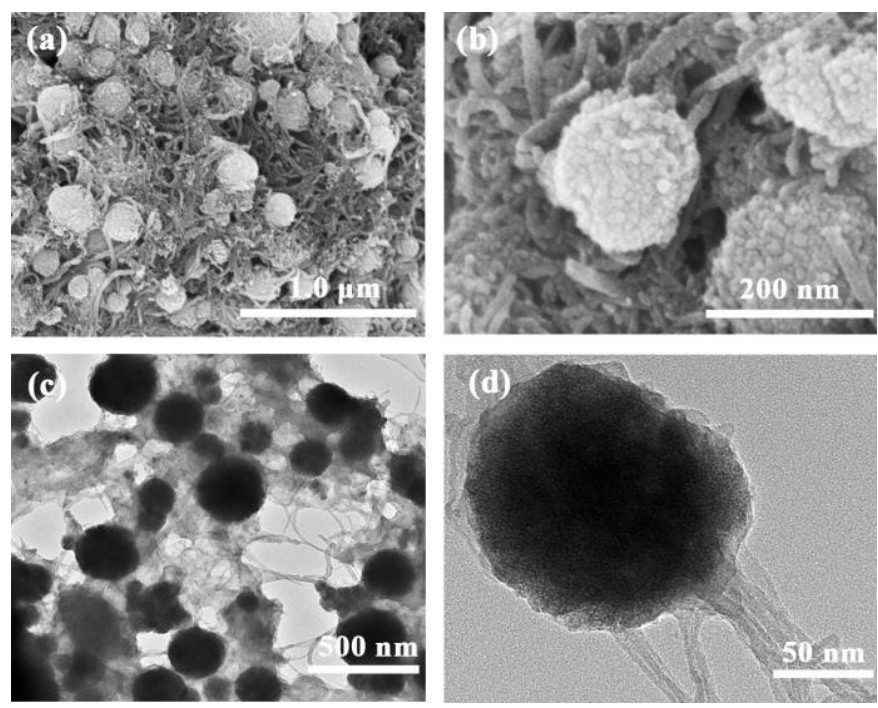

Figure S11. (a-b) SEM and (c-d) TEM images at different magnifications showing the morphology of CNFO@CNT-2.
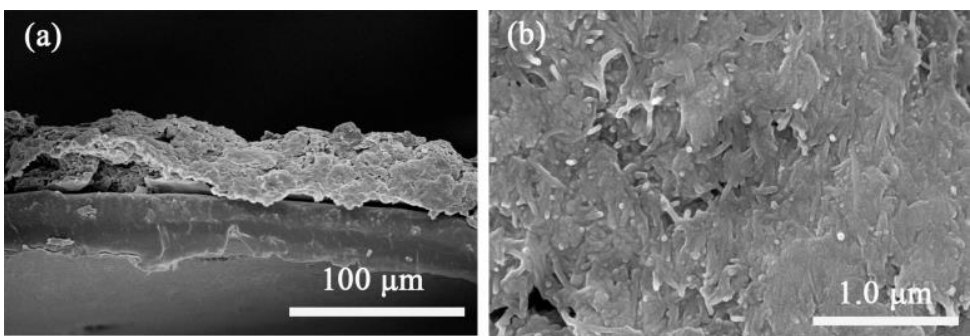

Figure S12. (a) Cross-sectional SEM and (b) the surface of CNFO@CNT/PP after 100 cycles at 1.0 C. 


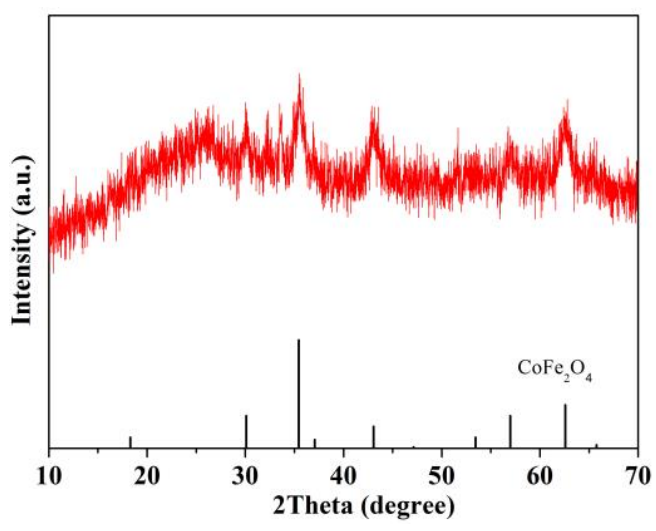

Figure S13. XRD pattern of the CNFO@CNT after 100 cycles at $1.0 \mathrm{C}$.
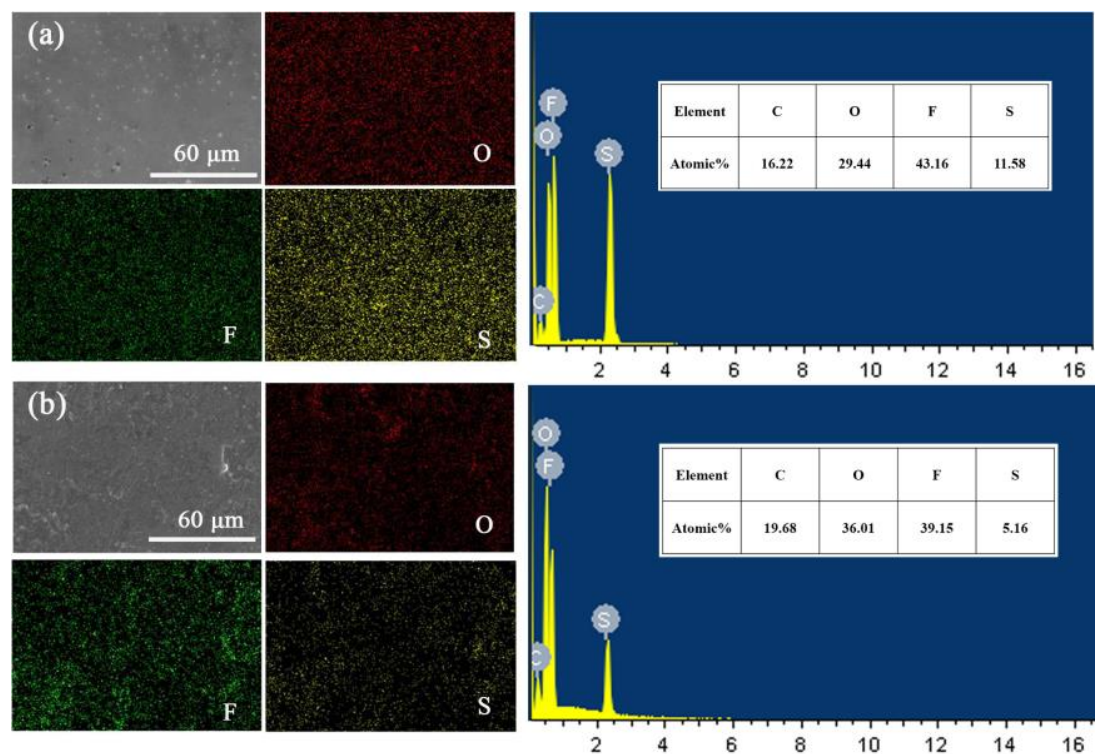

Figure S14. (a) SEM images and EDS spectras of Li-metal surface after cycling: Li-S batteries using (a) CNT interlayer PP and (b)CNFO@CNT interlayer. 
Table S1. Cycling performance of $\mathrm{Li}-\mathrm{S}$ batteries with different modification methods

\begin{tabular}{|c|c|c|c|c|}
\hline $\begin{array}{l}\text { Modified } \\
\text { method }\end{array}$ & $\begin{array}{l}\text { Initial capacity } \\
\qquad\left(\mathrm{mAh} \mathrm{g}^{-1}\right)\end{array}$ & $\begin{array}{c}\text { Capacity } \\
\text { retention }(100 \%)\end{array}$ & $\begin{array}{l}\text { Capacity } \\
\text { fading } \\
\text { cycle }^{-1}\end{array}$ & Reference \\
\hline CNFO@CNT & $897 \mathrm{mAh} \mathrm{g}^{-1}(2.0 \mathrm{C})$ & $84 \%$ (250 cycles $)$ & $0.063 \%$ & This work \\
\hline TiO-C65 & $1047 \mathrm{mAh} \mathrm{g}^{-1}(2.0 \mathrm{C})$ & $51 \%$ (300 cycles) & $0.16 \%$ & $\operatorname{Ref}^{[2]}$ \\
\hline $\mathrm{SiO}_{2}$ & $937 \mathrm{mAh} \mathrm{g}^{-1}(0.2 \mathrm{C})$ & $64 \%$ (200 cycles $)$ & $0.18 \%$ & $\operatorname{Re} f^{[3]}$ \\
\hline $\begin{array}{c}\text { Black- } \\
\text { Phosphorus }\end{array}$ & $\begin{array}{l}930 \mathrm{mAh} \mathrm{g}^{-1}(0.4 \mathrm{~A} \\
\left.\mathrm{g}^{-1}\right)\end{array}$ & $86 \%$ (100 cycles $)$ & $0.14 \%$ & $\operatorname{Ref}^{[4]}$ \\
\hline $\mathrm{MoS}_{2}$ & $808 \mathrm{mAh} \mathrm{g}^{-1}(0.5 \mathrm{C})$ & $50 \%$ (600cycles) & $0.83 \%$ & $\operatorname{Ref}^{[5]}$ \\
\hline MCNT@PEG & $998 \mathrm{mAh} \mathrm{g}^{-1}(0.5 \mathrm{C})$ & $49 \%$ (500 cycles $)$ & $0.10 \%$ & $\operatorname{Ref}^{[6]}$ \\
\hline $\mathrm{MnO}_{2}$ & $855 \mathrm{mAh} \mathrm{g}^{-1}(0.5 \mathrm{C})$ & $71 \%$ (500 cycles $)$ & $0.059 \%$ & $\operatorname{Ref}^{[7]}$ \\
\hline
\end{tabular}

\section{REFERENCES}

[1] Fan Q, Liu W, Weng Z, Sun Y.Wang H. Ternary Hybrid Material for High-Performance Lithium-Sulfur Battery[J]. $J$. Am. Chem. Soc. 2015, 137, 12946-12953.

[2] Xu G, Yan Q, Wang S, Kushima A, Bai P, Liu K, Zhang X, Tang Z.Li J. A thin multifunctional coating on a separator improves the cyclability and safety of lithium sulfur batteries[J]. Chem. Sci. 2017, 8, 6619-6625.

[3] Li J, Huang Y, Zhang S, Jia W, Wang X, Guo Y, Jia D.Wang L. Decoration of Silica Nanoparticles on Polypropylene Separator for Lithium-Sulfur Batteries[J]. ACS Appl. Mater. Interfaces 2017, 9, 7499-7504.

[4] Sun J, Sun Y, Pasta M, Zhou G, Li Y, Liu W, Xiong F.Cui Y. Entrapment of Polysulfides by a Black-PhosphorusModified Separator for Lithium-Sulfur Batteries[J]. Adv. Mater. 2016, 28, 9797-9803.

[5] Ghazi Z A, He X, Khattak A M, Khan N A, Liang B, Iqbal A, Wang J, Sin H, Li L.Tang Z. MoS $/$ Celgard Separator as Efficient Polysulfide Barrier for Long-Life Lithium-Sulfur Batteries[J]. Adv. Mater. 2017, 29, 1606817-1606823.

[6] Wang G C, Lai Y Q, Zhang Z A, Li J.Zhang Z Y. Enhanced rate capability and cycle stability of lithium-sulfur batteries with a bifunctional MCNT@PEG-modified separator[J]. J. Mater. Chem. A 2015, 3, 7139-7144. 
[7] Song X, Chen G, Wang S, Huang Y, Jiang Z, Ding L X.Wang H. Self-Assembled Close-Packed MnO2 Nanoparticles Anchored on a Polyethylene Separator for Lithium-Sulfur Batteries[J]. ACS Appl. Mater. Interfaces 2018, 10, $26274-26282$. 